\title{
A 1D-CNN Based Deep Learning Technique for Sleep Apnea Detection in IoT Sensors
}

\author{
Arlene John*, Barry Cardiff ${ }^{\dagger}$, Deepu John ${ }^{\dagger}$ \\ ${ }^{*}$ University College Dublin, Ireland \\ Email: *arlene.john@ucdconnect.ie, ${ }^{\dagger}\{$ barry.cardiff, deepu.john $\} @$ ucd.ie
}

\begin{abstract}
Internet of Things (IoT) enabled wearable sensors for health monitoring are widely used to reduce the cost of personal healthcare and improve quality of life. The sleep apneahypopnea syndrome, characterized by the abnormal reduction or pause in breathing, greatly affects the quality of sleep of an individual. This paper introduces a novel method for apnea detection (pause in breathing) from electrocardiogram (ECG) signals obtained from wearable devices. The novelty stems from the high resolution of apnea detection on a second-by-second basis, and this is achieved using a 1-dimensional convolutional neural network for feature extraction and detection of sleep apnea events. The proposed method exhibits an accuracy of $\mathbf{9 9 . 5 6 \%}$ and a sensitivity of $\mathbf{9 6 . 0 5 \%}$. This model outperforms several lower resolution state-of-the-art apnea detection methods. The complexity of the proposed model is analyzed. We also analyze the feasibility of model pruning and binarization to reduce the resource requirements on a wearable IoT device. The pruned model with $80 \%$ sparsity exhibited an accuracy of $97.34 \%$ and a sensitivity of $86.48 \%$. The binarized model exhibited an accuracy of $75.59 \%$ and sensitivity of $63.23 \%$. The performance of low complexity patient-specific models derived from the generic model is also studied to analyze the feasibility of retraining existing models to fit patient-specific requirements. The patient-specific models on average exhibited an accuracy of $\mathbf{9 7 . 7 9 \%}$ and sensitivity of $\mathbf{9 2 . 2 3 \%}$. The source code for this work is made publicly available.

Index Terms-Sleep apnea detection, IoT Sensors, Electrocar-
\end{abstract} diogram, Convolutional neural networks

\section{INTRODUCTION}

Sleep apnea-hypopnea syndrome is the abnormal reduction or pause in breathing during sleeping and is a disorder that affects $10 \%$ of middle-aged adults [1]. Sleep apnea can lead to neurological arousal affecting the quality of sleep and leading to daytime sleepiness, and fatigue [2]. In apnea, there is a complete pause in breathing, while in the case of hypopnea, there is a reduction in airflow characterized by a drop in oxygen saturation for at least 10 seconds [2]. Traditionally sleep-related disorders are diagnosed using overnight polysomnography under the supervision of a clinician. Recording polysomnograms for evaluation is costly and is not comfortable for the patient. Therefore, the development of a non-intrusive and automatic sleep-apnea detection method is of paramount importance.

Electrocardiogram (ECG) is a record of the electrical activity of the heart. In this work, we propose a sleep apnea detection technique using ECG, as it is possible to acquire

This work is supported by the Irish Research Council under the New Foundations Scheme. high quality ECG signals using non-invasive IoT wearable sensors [3]-[5]. Sleep apnea detection methods based on ECG signals, ECG derived respiration (EDR) and heart rate variability (HRV) have been proposed and could detect sleep apnea occurrence during a minute [2], [6]-[8], and occurrences in a 30-second window [9]. A systematic review of deep-learning-based methods for sleep apnea detection was carried out in [10]. We found that most studies on sleep-apnea detection using deep learning methods have a resolution of 1 minute or 30 seconds ie., inferences are made on a minute-by-minute basis or for a window of 30 seconds [11]-[14]. The highest resolution for sleep apnea detection from ECG signals (every $10 \mathrm{~s}$ ) was studied by Urtnasan et al. [11]. This leads to the contributions of this work:

1. Development of a second-by-second sleep apnea detection method, which is at a higher resolution compared to state-ofthe-art methods.

2. Development of a 1-dimensional convolutional neural network (1D-CNN), thereby reducing the need for feature extraction stages.

3. Complexity optimization of the developed 1D-CNN model using network pruning methods and binarization, as well as the development of patient-specific models to fit patientspecific requirements and achieve complexity reduction.

4. Performance and complexity analysis of the proposed methods is also discussed 1

\section{Methodology}

\section{A. Method Outline}

This paper proposes a method for sleep apnea detection on a per-second basis. This is achieved by considering a single window of 11 seconds containing the second of interest and using a 1D-CNN for classification. The window size is fixed at 11 seconds because an apnea event is classified as sleep apnea if the patient does not breathe for at least 10 seconds. For this, overlapping windows of 11 seconds with 10 seconds of overlap is generated. A window is assigned the label (apnea/nonapnea) depending on whether the $2^{\text {nd }}$ second in that window is apneic or non-apneic. No filtering or signal processing is carried out prior to the $1 \mathrm{D}-\mathrm{CNN}$ stage.

\footnotetext{
${ }^{1}$ Code and models available at https://github.com/arlenejohn/CNN_sleep_apnea
} 


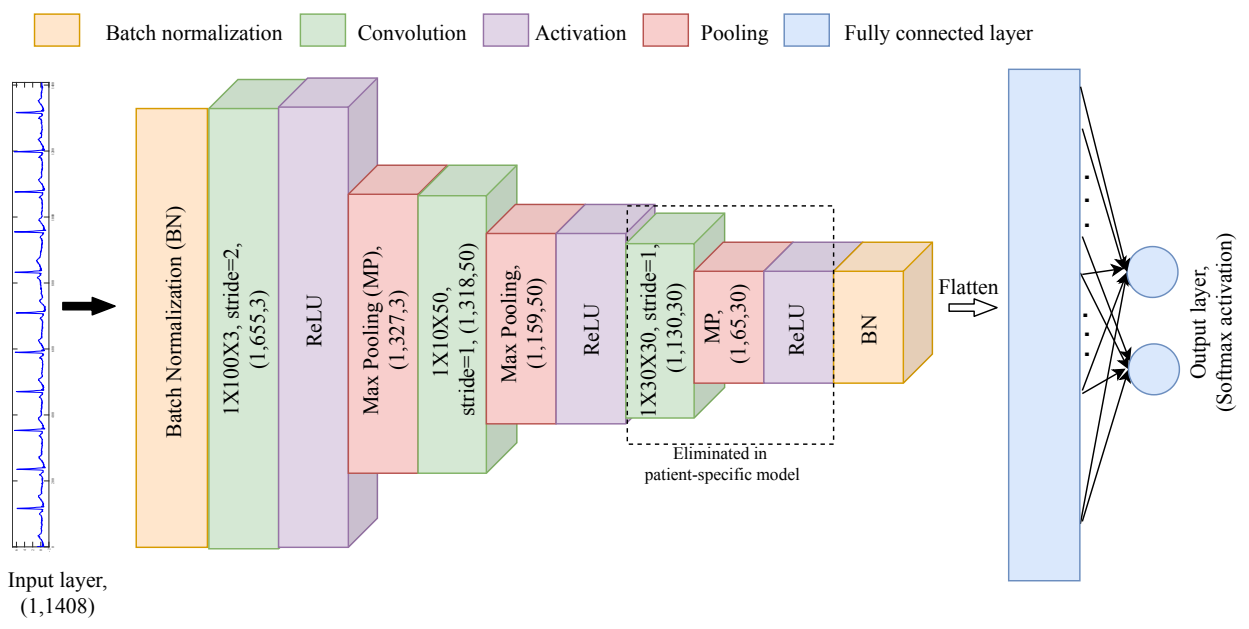

Fig. 1. The 1D-CNN model proposed for sleep apnea detection.

\section{B. Dataset}

In this paper, the UCD St. Vincent's University Hospital's sleep apnea database containing polysomnogram records from 25 patients is used [15]. We use the ECG signals sampled at $128 \mathrm{~Hz}$ for our study. The sleep seconds were marked as apneic or non-apneic based on the labels provided by the sleep experts. The dataset is split into training, validation, and test sets in the ratio of $8: 1: 1$. The training and validation set are balanced by oversampling the minority class (sleep apnea events). All the patient records, except the ones without any apnea events (ucddb008, ucddb011, ucddb013, and ucddb018), were used.

\section{C. $1 D-C N N$}

The 1D-CNN has 1408 (128 samples per second) nodes in the input layer. The samples are standard normalized prior to the input layer and the input layer is followed by batchnormalization stage. The $1 \mathrm{D}-\mathrm{CNN}$ uses 3 convolution layers with 3,50 , and 30 filters of size 100,10 , and 30 , respectively. The stride of the first convolution layer of 3 filters was set to 2 , while the stride was maintained as 1 for the other two convolution layers. The flow diagram of the neural network is shown in Fig. 1. In the model, all pooling layers use the maximum pooling method, and all activations use the ReLU activation function. The fully connected layers implement weight dropout with a probability of 0.25 during training. The model optimization used binary cross-entropy as the loss function with the ADAM optimizer. The learning rate was fixed as 0.001 , and the softmax activation function was applied to the output layer. The model considered has a total of 50,909 parameters. We can refer to this model as M1.

\section{Pruning}

A popular approach for reducing resource requirements at test time is pruning, which entails systematically removing parameters from an existing network [16]. In this work, we carry out weight-pruning ie., magnitude-based weight pruning to gradually zero out model weights during the training process to achieve model sparsity. This brings about improvements in model compression and is therefore suitable in resourceconstrained environments. We use the model M1 and attempt to sparsify the model with sparsity starting at $50 \%$ and slowly increase it upto the desired final sparsity level of $80 \%$ over 50 epochs. We refer to the pruned model as M2.

\section{E. Binarized $C N N$}

A method to develop neural networks with binary weights and activations at run-time was proposed in [17]. Binarized kernel elements and weights reduce memory size and accesses and substantially improves power-efficiency. In this work, we binarize the kernel parameters in the convolution layers and the weights in the fully connected layers of the model. The activations and batch-normalization layers are not binarized. This model has 50,824 parameters as we do not use bias terms in this model. We refer to the binarized model as M3.

\section{F. Generating Patient-Specific Models}

An approach to improve patient-specific inferences is using patient-specific models derived from the generic model discussed in Section III-C The advantage of this method is that the number of model parameters can be reduced by eliminating a few kernels or eliminating an entire layer. In this paper, we attempt to remove the last convolution layer of 30 filters, the subsequent max-pooling, and activation layers completely as shown in Fig. 1. We maintain the initials weights and parameters in the two convolution layers that are obtained from the generic model and then retrain the full model with the patient-specific training data. This brings down the total number of model parameters to 17,959 . We refer to this patient-specific model as M4.

\section{RESULTS}

\section{A. Performance Analysis}

The model M1 was trained over the full training set and simultaneously validated on the validation set for each epoch, where a true positive stands for an accurately detected apnea 

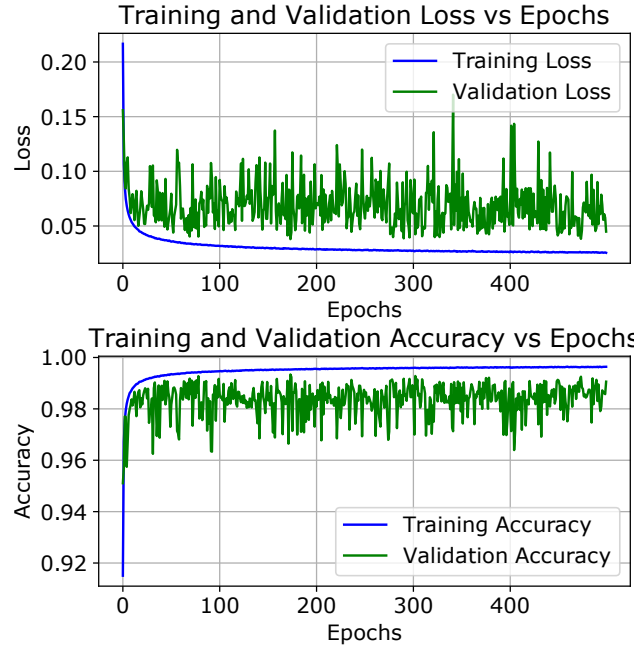

Fig. 2. (Top) Training and validation loss vs epochs and (bottom) training and validation accuracy vs epochs during training of model M1.

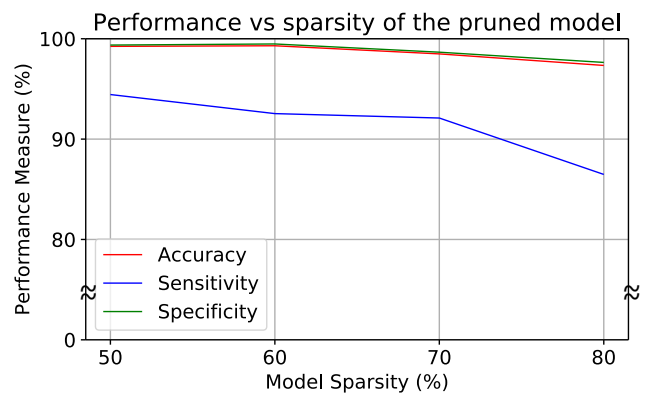

Fig. 3. Performance of the pruned model on the test set when sparsity is increased from $50 \%$ to $80 \%$.

TABLE I

PERFORMANCE OF THE PATIENT-SPECIFIC MODEL, M4

\begin{tabular}{|c|c|c|c|}
\hline Patient & Accuracy (\%) & Sensitivity (\%) & Specificity (\%) \\
\hline ucddb002 & 99.91 & 93.75 & 99.96 \\
\hline ucddb003 & 96.94 & 93.42 & 97.04 \\
\hline ucddb005 & 100.00 & 100.00 & 100.00 \\
\hline ucddb006 & 96.39 & 84.52 & 96.83 \\
\hline ucddb007 & 98.77 & 85.71 & 96.30 \\
\hline ucddb009 & 99.68 & 92.31 & 99.75 \\
\hline ucddb010 & 100.00 & 100.00 & 100.00 \\
\hline ucddb012 & 97.44 & 90.36 & 97.69 \\
\hline ucddb014 & 99.96 & 100.00 & 99.96 \\
\hline ucddb015 & 99.93 & 100.00 & 99.93 \\
\hline ucddb017 & 99.96 & 100.00 & 99.96 \\
\hline ucddb019 & 99.06 & 85.37 & 99.28 \\
\hline ucddb020 & 99.91 & 100.00 & 99.99 \\
\hline ucddb021 & 99.85 & 92.86 & 99.89 \\
\hline ucddb022 & 100.00 & 100.00 & 100.00 \\
\hline ucddb023 & 99.96 & 100.00 & 99.96 \\
\hline ucddb024 & 98.97 & 90.70 & 99.10 \\
\hline ucddb025 & 81.96 & 78.78 & 83.00 \\
\hline ucddb026 & 99.48 & 85.71 & 99.68 \\
\hline ucddb027 & 88.68 & 84.29 & 89.16 \\
\hline ucddb028 & 96.84 & 79.07 & 97.64 \\
\hline Average & $\mathbf{9 7 . 7 9}$ & $\mathbf{9 2 . 2 3}$ & $\mathbf{9 7 . 8 6}$ \\
\hline & & & \\
\hline
\end{tabular}

event. Validation callback was used to determine the best weights during training based on which set of weights exhibited the highest validation accuracy. The training and validation loss against each epoch and the training and validation

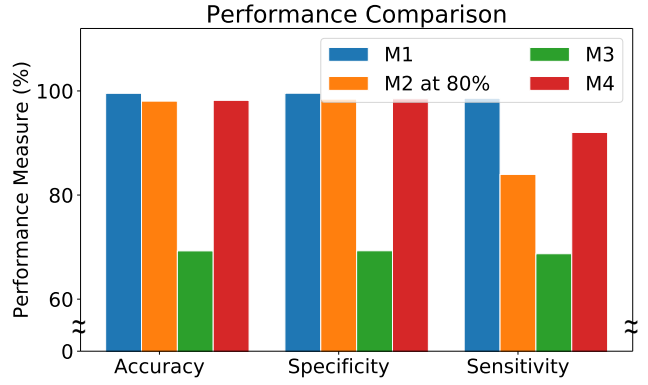

Fig. 4. Comparison bar plot of Accuracy, Specificity, and Sensitivity of the three models M1, M2, M3, and M4.

TABLE II

Average Performance of the three models M1, M2, M3, AND M4 IN TERMS OF ACCURACY, SENSITIVITY, AND SPECIFICITY

\begin{tabular}{|c|c|c|c|}
\hline Model & Accuracy (\%) & Sensitivity (\%) & Specificity (\%) \\
\hline M1 & 99.56 & 96.05 & 99.66 \\
\hline M2 at $80 \%$ & 97.34 & 86.48 & 97.64 \\
\hline M2 at 70\% & 98.49 & 92.10 & 98.66 \\
\hline M2 at 60\% & 99.30 & 92.54 & 99.48 \\
\hline M2 at 50\% & 99.24 & 94.44 & 99.37 \\
\hline M3 & 75.59 & 63.23 & 75.92 \\
\hline M4 & 97.79 & 92.23 & 97.86 \\
\hline
\end{tabular}

accuracy against each epoch is shown in Fig. 2. The model M1 exhibited an accuracy of $99.56 \%$, a specificity of $99.66 \%$, and a sensitivity of $96.05 \%$ on the test set consisting of data from all the patients.

For the pruned model, we study the performance when the final model sparsity is at $50 \%, 60 \%, 70 \%$, and $80 \%$. The pruning method reduces the model complexity by increasing the sparsity of the convolutional layers and the fully connected layer to the desired level of sparsity. The performance of the pruned model in terms of accuracy, specificity, and sensitivity over varying sparsity levels is shown in Fig. 3. As expected, the performance drops with an increase in sparsity levels, and therefore a tradeoff between power/resource consumption and performance is required to fix the optimal sparsity level. The pruned model M2 exhibited an accuracy of $97.34 \%$, a specificity of $97.64 \%$, and a sensitivity of $86.48 \%$ on the test set consisting of data from all the patients at the highest sparsity level of $80 \%$.

The binarized model M3 was trained over the full training set and simultaneously validated on the validation set for each epoch. The model M3 exhibited an accuracy of $75.59 \%$, a specificity of $75.92 \%$, and a sensitivity of $63.23 \%$ on the test set consisting of data from all the patients. When compared to the models M1 and M2, the performance of the binarized model is poor. Further investigations into various combinations of binarized layers and normal layers needs to be carried out to achieve the desired performance levels with improvements in power-efficiency.

M4 was obtained by dropping the last convolution, pooling, and activation layers, and this model was retrained for each patient with the training data taken from just that one patient to generate patient-specific models. The performance for each 
TABLE III

COMPUTATIONAL COMPLEXITY AND ENERGY CONSUMPTION OF THE MODELS DURING PREDICTION

\begin{tabular}{|c|c|c|c|c|}
\hline Model & Net Parameters & Multiplications & Additions & Energy $(\mu \mathbf{J})$ \\
\hline M1 & 50909 & 6534116 & 6546647 & 2.55 \\
\hline M2 at $80 \%$ & 10349 & 1312232 & 1324763 & 0.52 \\
\hline M2 at 70\% & 14247 & 1964936 & 1977467 & 0.77 \\
\hline M2 at $60 \%$ & 20489 & 2617676 & 2630207 & 1.03 \\
\hline M2 at 50\% & 25559 & 3270416 & 3289663 & 1.28 \\
\hline M3 & 50824 & 4766 & 6301530 & 0.13 \\
\hline M4 & 17959 & 708116 & 717197 & 0.28 \\
\hline
\end{tabular}

patient-specific model tested on the test set derived from that patient's record is shown in Table II The results over all the patient-specific models are found to have an average accuracy of $97.79 \%$, a specificity of $97.86 \%$, and a sensitivity of $92.23 \%$.

The performance of the four models (M2 at 80\%) in terms of accuracy, specificity, and sensitivity are compared in Fig. 4 The performance parameters of all the models are detailed in Table II. From the results, it can be concluded that the models M1, M2, and M4 are suitable for second-by-second apnea detection, and $\mathrm{M} 3$ requires further investigation.

\section{B. Complexity Calculation}

The model complexity was calculated in terms of the number of multiplications and additions required for sleep apnea detection per second [18]. Prediction with the model M1 requires 6534116 multiplications and 656647 additions. Prediction with the pruned model M2 requires the same number of multiplications and additions as M1. However, we can approximate the gains due to pruning by calculating the number of multiplications and additions with non-zero numbers. When M2 is $80 \%$ sparse compared to M1 in the convolutional layers and dense layers, we can approximately calculate the number of multiplications required as 1312232 and additions as 1325763. When the sparsity is $70 \%$, the number of multiplications required is 1964936, and additions are 1977467. When the model sparsity is $60 \%$, the model effectively requires 2617676 multiplications and 2630207 additions for prediction. 3270416 multiplications and 3289663 additions are required for prediction when the sparsity is $50 \%$. Prediction with the binarized model M3 requires a series of addition operations at the convolution layers and fully connected layer since the weights are either +1 or -1 . Therefore, prediction with model M3 requires 4766 multiplications and 6301530 additions. Prediction with the model M4 requires approximately 708116 multiplications and 717197 additions. The total energy consumption during prediction using M1 is found to be $2.55 \mu \mathrm{J}, \mathrm{M} 2$ at $80 \%$ sparsity to be $0.52 \mu \mathrm{J}$, M2 at $70 \%$ sparsity to be $0.77 \mu \mathrm{J}, \mathrm{M} 2$ at $60 \%$ sparsity to be 1.03 $\mu \mathrm{J}, \mathrm{M} 2$ at $50 \%$ sparsity to be $1.28 \mu \mathrm{J}$, M3 to be $0.13 \mu \mathrm{J}$, and M4 to be $0.28 \mu \mathrm{J}$. This is calculated by assuming that the energy required is $0.39 \mathrm{pJ}$ for a 16-bit by 16-bit multiplication accumulation (MAC) operation [19], [20], and approximately $20 \mathrm{fJ}$ for a 16-bit by 16-bit addition operation [21] in $28 \mathrm{~nm}$ FD-SOI technology. The computational complexity and energy consumption are discussed in Table III

The performance of the discussed 1D-CNN based sleep apnea detection model is compared against the performance of state-of-the-art sleep apnea detection algorithms in Table IV] It can be observed from Table IV that the proposed models M1, M2, and M4 exhibits the best performance in terms of accuracy and exhibits comparable performance in terms of sensitivity, at the highest resolution of 1 second.

\section{Conclusions}

In this paper, we explore the automatic sleep apnea detection problem from ECG signals for wearable IoT sensors. Unlike other works with detection resolutions as high as 1 minute, the developed system performs event detection on a secondby-second basis. A 1D-CNN is employed to learn the requisite features for event detection. We analyze 3 strategies to reduce the model size to make it suitable for resource-constrained environments and compare the performance amongst these. The proposed method achieved an accuracy of $99.56 \%$, the pruned model achieved an accuracy of $97.34 \%$, the binarized model exhibited an accuracy of $75.59 \%$, and the patientspecific models achieved an average accuracy of $97.79 \%$. Future studies would focus on the development of much smaller models through knowledge distillation by making use of the model discussed in this paper is another step. These models can be used for sleep apnea detection on a second-bysecond basis for any patient with minimal tuning. The work would also focus on analyzing the filters learned by the 1D$\mathrm{CNN}$ layers to explain the feature extraction process and its impact on the model performance.

TABLE IV

COMPARISON OF THE PERFORMANCE OF THE PROPOSED CNN MODEL FOR APNEA DETECTION WITH STATE-OF-THE-ART ECG RECORD BASED SLEEP APNEA DETECTION ALGORITHMS.

\begin{tabular}{|c|c|c|c|c|c|c|c|c|}
\hline Article & Urtnasan et al. 11 & Wang et al. 12 & Dey et al. 13 & Chen et al. 14 & \multicolumn{4}{|c|}{ This work } \\
\hline Dataset & $\begin{array}{l}\text { Samsung Medical } \\
\text { Center dataset }\end{array}$ & $\begin{array}{l}\text { UCD apnea } \\
\text { database }\end{array}$ & $\begin{array}{l}\text { Physionet Apnea } \\
\text { ECG database }\end{array}$ & $\begin{array}{c}\text { Physionet Apnea ECG database } \\
\text { and UCD apnea database }\end{array}$ & \multicolumn{4}{|c|}{$\begin{array}{c}\text { UCD apnea } \\
\text { database }\end{array}$} \\
\hline Resolution & 10 seconds & 1 minute & 1 minute & $\begin{array}{l}\text { Variable resolution } \\
\text { upwards of } 30 \text { seconds }\end{array}$ & \multicolumn{4}{|c|}{1 second } \\
\hline \multirow{2}{*}{ Method } & \multirow{2}{*}{$\mathrm{CNN}, 6$ layers } & \multirow{2}{*}{$\mathrm{CNN}, 2$ layers } & \multirow{2}{*}{$\mathrm{CNN}, 3$ layers } & \multirow{2}{*}{$\begin{array}{l}\text { Support vector machines } \\
\text { with selected features }\end{array}$} & \multicolumn{4}{|c|}{ 1D-CNN } \\
\hline & & & & & M1 & $\begin{array}{c}\text { M2 } \\
(80 \%)\end{array}$ & M3 & M4 \\
\hline Accuracy & $96.00 \%$ & $71.80 \%$ & $98.91 \%$ & $92.87 \%$ & $99.56 \%$ & $\mathbf{9 7 . 3 4} \%$ & $75.59 \%$ & $97.79 \%$ \\
\hline Sensitivity & $96.00 \%$ & $26.6 \%$ & $97.82 \%$ & $97.41 \%$ & $96.05 \%$ & $\mathbf{8 6 . 4 8} \%$ & $63.23 \%$ & $\mathbf{9 2 . 2 3} \%$ \\
\hline
\end{tabular}




\section{REFERENCES}

[1] P. E. Peppard et al., "Increased prevalence of sleep-disordered breathing in adults." American journal of epidemiology, vol. 177, no. 0, pp. 10061014, May 2013.

[2] B. Xie and H. Minn, "Real-time sleep apnea detection by classifier combination," IEEE Transactions on Information Technology in Biomedicine, vol. 16, no. 3, pp. 469-477, 2012.

[3] D. L. T. Wong et al., "An integrated wearable wireless vital signs biosensor for continuous inpatient monitoring," IEEE Sensors Journal, vol. 20, no. 1, pp. 448-462, 2020.

[4] A. John, B. Cardiff, and D. John, "A generalized signal quality estimation method for IoT sensors," in 2020 IEEE International Symposium on Circuits and Systems (ISCAS), 2020, pp. 1-5.

[5] A. John et al., "An approximate binary classifier for data integrity assessment in IoT sensors," in 2020 27th IEEE International Conference on Electronics, Circuits and Systems (ICECS), 2020, pp. 1-4.

[6] C. Varon et al., "A novel algorithm for the automatic detection of sleep apnea from single-lead ECG," IEEE Transactions on Biomedical Engineering, vol. 62, no. 9, pp. 2269-2278, 2015.

[7] H. D. Nguyen et al., "An online sleep apnea detection method based on recurrence quantification analysis," IEEE Journal of Biomedical and Health Informatics, vol. 18, no. 4, pp. 1285-1293, 2014.

[8] R. Atri and M. Mohebbi, "Obstructive sleep apnea detection using spectrum and bispectrum analysis of single-lead ECG signal," Physiological Measurements, vol. 36, no. 9, pp. 1963-1980, 2015.

[9] B. Sulistyo, N. Surantha, and S. M. Isa, "Sleep apnea identification using HRV features of ECG signals," International Journal of Electrical and Computer Engineering (IJECE), vol. 8, p. 3940, 102018.

[10] S. S. Mostafa, F. Mendonça, A. G. Ravelo-García, and F. Morgado-Dias, "A systematic review of detecting sleep apnea using deep learning," Sensors, vol. 19, no. 22, 2019.

[11] E. Urtnasan et al., "Automated detection of obstructive sleep apnea events from a single-lead electrocardiogram using a convolutional neural network," J. Med. Syst., vol. 42, no. 6, p. 1-8, Jun. 2018.

[12] T. Wang et al., "Sleep apnea detection from a single-lead ECG signal with automatic feature-extraction through a modified LeNet-5 convolutional neural network," PeerJ, vol. 7, p. e7731, Sep. 2019.

[13] D. Dey, S. Chaudhuri, and S. Munshi, "Obstructive sleep apnoea detection using convolutional neural network based deep learning framework," Biomedical Engineering Letters, vol. 8, pp. 95-100, Dec 2017.

[14] L. Chen, X. Zhang, and C. Song, "An automatic screening approach for obstructive sleep apnea diagnosis based on single-lead electrocardiogram," IEEE Transactions on Automation Science and Engineering, vol. 12, no. 1, pp. 106-115, 2015.

[15] "St. Vincents University Hospital/ University College Dublin sleep apnea database," 2011. [Online]. Available: http://physionet.org/physiobank/ database/ucddb/

[16] D. Blalock et al., "What is the state of neural network pruning?" 2020

[17] M. Courbariaux et al., "Binarized neural networks: Training deep neural networks with weights and activations constrained to +1 or $-1, " 2016$.

[18] A. John et al., "Binary classifiers for data integrity detection in wearable IoT edge devices," IEEE Open Journal of Circuits and Systems, vol. 1, pp. 88-99, 2020.

[19] H. Reyserhove, N. Reynders, and W. Dehaene, "Ultra-low voltage datapath blocks in 28nm UTBB FD-SOI," in 2014 IEEE Asian SolidState Circuits Conference (A-SSCC), Nov 2014, pp. 49-52.

[20] R. Taco et al., "An 88-fJ/40-MHz [0.4 V]-0.61-pJ/1-GHz [0.9 V] DualMode Logic $8 \times 8$ bit Multiplier Accumulator With a Self-Adjustment Mechanism in 28-nm FD-SOI," IEEE Journal of Solid-State Circuits, vol. 54, no. 2, pp. 560-568, Feb 2019.

[21] R. Taco et al., "Evaluation of Dual Mode Logic in 28nm FD-SOI technology," in 2017 IEEE International Symposium on Circuits and Systems (ISCAS), 2017, pp. 1-4. 